\title{
Promotion of Egg and Eggshell Powder Consumption on the Nutritional Status of Young Children in Ethiopia
} Anteneh Omer ${ }^{1}$, Demmelash Mulualem ${ }^{2}$, Henry Classen ${ }^{3}$, Hassan Vatanparast ${ }^{4}$, Susan J. Whiting ${ }^{5 *}$

${ }^{1}$ School of Nutrition, Food Science and Technology, College of Agriculture, Hawassa University, Ethiopia

${ }^{2}$ School of Nutrition, Food Science and Technology, College of Agriculture, Hawassa University, Ethiopia

${ }^{3}$ College of Agriculture and Bioresources, University of Saskatchewan, Canada

${ }^{4}$ College of Pharmacy and Nutrition, University of Saskatchewan, Canada

${ }^{5}$ College of Pharmacy and Nutrition, University of Saskatchewan, Canada

\begin{abstract}
Introduction: Complementary feeding presents a challenge for very young children in Ethiopia as these foods are generally thin cereals providing little protein and micronutrients. We tested whether an egg-a-day and eggshell powder (ESP) providing calcium (Ca) will improve nutritional status of young children in a 6-month randomized controlled cluster trial in Southern Ethiopia.

Methods: Study sites were randomly selected kebeles (villages) and assigned to Intervention and Control groups. All eligible children 6-12 months were enrolled. Each child in Intervention kebeles received a gift of two egg laying chickens $(\mathrm{n}=122)$; families received education on poultry production and consumption of egg. ESP (500 mg Ca) was given to children at $\geq 12$ mo. The Control group $(n=128)$ had standard nutrition education. Baseline and endline outcome measures included knowledge, attitude, and practice (KAP) of caregivers on the feeding of egg and ESP, anthropometry, child dietary intake frequencies, egg sensitivities, hemoglobin, and morbidity symptoms.
\end{abstract}

Results: Egg and ESP consumption in Intervention children averaged 17 days/month compared to $<1$ in Controls. KAP of mothers improved only in the intervention group ( $p<0.001)$. No true egg allergy occurred, however, $7.5 \%$ of Intervention children were sensitive to eggs. Linear regression analysis showed the egg+ESP intervention increased weight-for-age z-score by 0.24 (95\% CI, 0.11-0.37) and reduced underweight (RR, 0.35; 95\% CI, 0.16-0.74). Stunting prevalence increased in both groups, but by $28 \%$ less in the treatment group (RR, 0.72; 95\% CI, 0.53-0.98) compared with control. Mean hemoglobin increased and anemia decreased in both groups, however, much greater anemia reduction $[52 \%(\mathrm{RR}, 0.48 ; 95 \% \mathrm{CI}, 0.24-0.96)]$ was seen in the treatment group.

Conclusion: Promotion of egg and ESP consumption with poultry intervention significantly improved the nutritional status of young children, demonstrating the potential of an integrated approach to contribute to the country's target to reduce malnutrition.

Keywords: Homestead chicken, Eggshell, Egg-a-day, Under 2 children, Cluster randomized trial

\section{Introduction}

From 2000 to 2016, Ethiopia recorded a consistent declining trend of stunting and underweight from $58 \%$ to $38 \%$ and $41 \%$ to $24 \%$, the decrease of $1.25 \%$ and $1.063 \%$ per year, respectively. Yet stunting and underweight remain significant public health challenges of Ethiopia [1]. Wasting showed little decrease in the past sixteen years. To the contrary, anaemia has been reported to have increased from $44 \%$ in 2011 to $57 \%$ in 2016, presenting a further challenge to combatting malnutrition [1].
Poor feeding practices are one of the major factors contributing to under nutrition in Ethiopia.

Corresponding Author: Susan J Whiting, College of Pharmacy and Nutrition, University of Saskatchewan 104 Clinic Place, Saskatoon SK Canada E-mail: susan.whiting@usask.ca Received date: April 14, 2019; Accepted date: April 22, 2019; Published date: April 23, 2019.

DOI: https://doi.org/10.31546/IJFSNR.1004 
Complementary foods are mainly cereal-based with low protein, fat and micronutrient density. The Ethiopian National Food Consumption Survey reported carbohydrates contribute more than $67 \%$ of the total energy intake of under three children while proteins and fat contribute $10 \%$ and $23 \%$ respectively. It also noted that about $52 \%$ had inadequate protein intake [2].

It is recommended that infants and young children should be fed on four or more of the seven food groups so that the child will have high likelihood of consuming at least one animal source food (ASF). In Ethiopia, consumption of flesh foods, milk and eggs has been reported to be very low among children $6-23$ months with $8 \%, 15.6 \%$ and $17 \%$ respectively [1]. Eggs are foods having high quality protein containing all the essential amino acids in ample quantities. In addition, they are rich sources of the B vitamins, choline, vitamin A and micro minerals including selenium, iron, and zinc [3]. Another advantage of eggs arises from their shell which is rich in bioavailable calcium [4]. Dry eggshells can be finely powdered very easily using common kitchen appliances and added to foods without changing colour, taste and texture of the food [5].

Eggs are relatively cheap ASFs and can be made easily available through homestead poultry. Studies of small-scale poultry integrated with nutrition education, however, have not consistently found increased consumption of eggs by infants and young children [6,7]. In these studies, caregivers gave priority to sell the eggs and birds, to invest more on their poultry enterprise, rather than feeding eggs to the children. In contrast, an intervention in Ecuador gave eggs rather than chicken, and reported a significant reduction of stunting and underweight by $47 \%$ and $74 \%$ respectively, showing the potential effect of an egg a day in improving growth of infants and young children [8]. Thus, there is little evidence of a homestead chicken intervention on child growth.

We previously reported on how an integrated intervention involving a chicken gift ceremony to children, accompanied by nutrition education and agriculture extension support in the community, led to increased egg consumption of young children and increased poultry production in intervention than control communities [9]. Our primary objective in this follow-up paper is to describe nutritional and hemoglobin status, and rates of morbidity among infants and young children in that 6-month study in rural Ethiopia.

\section{Methods}

\section{Study Design, Area and Population}

Details of the study area, design and methods have been described elsewhere [9].
Briefly, it was an interventional study design of cluster randomized controlled community trial conducted from May-Nov 2016 in Halaba, a food insecure district in the Southern Region of Ethiopia. Livelihood mainly depends on rain-fed agriculture producing maize, sorghum, millet, haricot bean, chilli and potato with livestock production in which poultry ranks fourth next to cow, ox and donkeys. The study was conducted in Debeso, Yeye, Shewako Halaba, Yambo, Aymele and Andegna Tuka kebeles (villages). The first three locations were randomly allocated in the intervention arm and the remaining were in the control. All mother (caregiver)-infant pairs who lived in the study sites for at least 6 months before the time of the study were recruited targeting children aged 6-12 month. Eligibility criteria were as follows: singleton birth and healthy without moderate/severe acute malnutrition or not enrolled in any nutritional therapeutic programs as well as no known egg allergy.

The sample size was determined as 130 in each arm taking cluster randomization, $10 \%$ loss to follow-up, $\alpha=.05,1-\beta=0.80$ and effect size of 0.35 in consideration of enough power of detection of an increase in weight for age based on literature [10]. Informed consent was obtained from each caregiver before the baseline survey for confirmation of willingness to participate in the study. Ethical clearance was obtained from University of Saskatchewan, Canada and Hawassa University, Ethiopia. The trial was registered at clinicaltrials.org with an identifier NCT02791100.

Halaba special Woreda is divided into ten (clusters) catchment areas according to the structure used by the health sector. Two catchment areas namely; Guba and Tuka, were selected by lottery method and randomly allocated to the intervention and the control arm respectively. The inclusion of kebeles from a given catchment was made randomly by lottery method until the desired sample size was acquired. Care was taken during inclusion of kebeles that a given study kebele in one catchment did not share common boundary and market with another study kebele in the other catchment in order to minimize information contamination between the intervention and control groups. Since cluster randomization is preferred over individual-level randomization to minimize contamination, all target children who fulfilled the requirement in a given selected kebele were enrolled in the study. 
Targets were registered using family folders in health posts followed by house to house check-ups for any missed child and double checking of ages with each caregiver.

\section{Data Collection Procedures and Instruments}

Dietary, morbidity, and poultry production assessments were carried out at baseline and endline by trained data collectors blind of group assignment and monthly by a nutritionist and animal health experts. Morbidities were assessed focusing on highly prevalent diseases in the study area including diarrhea, respiratory and malaria as well as egg allergy symptoms.

Anthropometry measurements of weight, length and MUAC were taken at baseline, monthly (weight and MUAC only) and endline. A digital scale of Seca Model 874 (Seca GmbH \& Co KG) with the mother-child tare feature used for measuring weight. Two measures of weight were taken and when differed by $0.03 \mathrm{~kg}$ or more, a third measurement was taken and averaged with the closest measure. Subjects were measured twice for length using Shorr measuring board to the nearest $0.1 \mathrm{~cm}$. When measures were differed by more than $0.3 \mathrm{~cm}$ or more, a third measure was taken and averaged with the closest one. The left arm of the young children was used to measure MUAC using arm circumference insertion tapes, to the nearest $0.1 \mathrm{~cm}$. The measurement was obtained with the caregiver seated and holding the young child on her lap. Hemoglobin tests were carried out using HemoCue $\mathrm{Hb} 301$ (HemoCue AB, Ängelholm, Sweden). Standard operating procedures and instructions were strictly followed during blood collection and the testing. Values were adjusted for altitude [11].

The intervention has been described previously [9]. In brief, it had two major components: capacity building and chicken gifting, and both were community-based, involving experts at district and community level, particularly health and agriculture extension workers and health development team leaders, as well as caregivers. With a goal of increasing egg intake, infants and young children were gifted two local chickens vaccinated for Newcastle disease in an innovative community approach named 'Chicken Gift Ceremony' where caregivers promised in front of religious and community leaders to add at least two more, replace if die and not to sell nor share the eggs or chickens [9].

Moreover, each caregiver received counseling monthly at health post using BCC materials developed for this study (colored laminated cards) promoting one egg and one measure (defined as half a cork top) eggshell powder (ESP) a day and environmentally safe poultry production. Nutrition messages focused on egg and ESP preparation and feeding while poultry messages emphasized four egg-laying hens per child, preparation of chicken shelter, environmental sanitation, and New Castle vaccination.
Hard-boiled and smashed egg was promoted in this study considering its easy preparation that requires only water and pot to boil and fork or spoon to smash. Breast milk was used to soften the smashed egg. The eggshells were collected, boiled, and sun dried so that they could be finely powdered using wooden pestle and mortar or local mill made of stone. ESP intake was promoted for children above one year as their calcium requirement increases at that age.

\section{Data Analysis}

WHO Anthro v3.2.2 was used for calculation of anthropometric indices of z-scores of length for age (LAZ, also called HAZ when a child was measured in standing position), WAZ and weight for height (WHZ). Stunting, underweight, and wasting were defined as LAZ, WAZ, and WHZ less than -2 standard deviations (SD) below median values respectively. Paired t-test was used to evaluate differences on outcomes before and after intervention. Independent two-sample t-tests were run to assess statistical differences on outcomes between intervention and control groups at baseline and end line. Trend of underweight and stunting rates was analyzed over the intervention period using visual inspection. Regression models adjusted for corresponding baseline values, age, sex of the child and other covariates were run to assess association and estimate effect size for continuous variables. Prevalence risk ratios (RR) were used as risk estimate to evaluate the effect of egg and ESP intake on underweight, stunting, and anemia. P-value $<0.05$ was considered significant. SPSS software (IBM SPSS Statistics, Version 20) was used for analysis.

\section{Results}

Out of 287 mother-child pairs who passed eligibility, 15 did not meet inclusion criteria. A total of 272 infants and young children were randomly allocated to the intervention $(n=140)$ and control $(n=132)$. Due to a death by fire accident and three relocations, four children were lost to follow-up only from the control arm (Figure 1). However, 18 children from the intervention were found to be sensitive to egg and excluded from analysis. As a result, the analysis was conducted for 250 mother-child pairs (122 in the intervention and 128 in the control group). 
Anteneh Omer et al.

Table 1: Baseline characteristics of study participants by trial groups

\begin{tabular}{|c|c|c|c|}
\hline Baseline Characteristics & & Intervention $(\mathrm{n}=122)$ & $\begin{array}{l}\text { Control } \\
(\mathrm{n}=128)\end{array}$ \\
\hline \multicolumn{4}{|l|}{ Maternal } \\
\hline \multirow{6}{*}{ Main occupation } & Mean (SD), y & $27.4(5.2)$ & $28.0(5.6)$ \\
\hline & Average age at first birth (SD) & $18.3(1.9)$ & $18.4(2.9)$ \\
\hline & Farmer, \% & $114(93.4)$ & $126(98.4)$ \\
\hline & Government employee, $\%$ & $2(1.6)$ & $1(0.8)$ \\
\hline & Petty trader, \% & $4(3.3)$ & $1(0.8)$ \\
\hline & No job, \% & $2(1.6)$ & $\mathbf{0}(\mathbf{0})$ \\
\hline \multirow[t]{3}{*}{ Education status } & Illiterate, $\%$ & $92(75.4)$ & $96(75.0)$ \\
\hline & Read and write, $\%$ & $3(2.5)$ & $\mathbf{0}(\mathbf{0})$ \\
\hline & Formal education, $\%$ & $27(22.1)$ & $32(25.0)$ \\
\hline \multicolumn{4}{|l|}{ Child } \\
\hline \multirow{5}{*}{$\begin{array}{l}\text { Age } \\
\text { up* }\end{array}$} & Mean (SD), mo & $9.3(1.9)$ & $9.8(1.9)$ \\
\hline & 6-8 months, $\%$ & $53(43.4)$ & $40(31.3)$ \\
\hline & 9-12 months, \% & $69(56.6)$ & $88(68.8)$ \\
\hline & Male, \% & $66(54.1)$ & $67(52.3)$ \\
\hline & Female, \% & $56(45.9)$ & $61(47.7)$ \\
\hline \multirow[t]{3}{*}{ Child birth order, \% } & First, \% & 17 (13.9) & $19(14.8)$ \\
\hline & Second, \% & $10(8.2)$ & $17(13.3)$ \\
\hline & Third or above, $\%$ & 95 (77.9) & 92 (71.9) \\
\hline \multirow{3}{*}{$\begin{array}{r}\text { Number of }<5 \text { children in } \\
\text { house hold }\end{array}$} & One, $\%$ & $31(25.4)$ & $32(25.0)$ \\
\hline & Two, \% & $71(58.2)$ & $78(60.9)$ \\
\hline & Three, \% & $20(16.4)$ & $18(14.1)$ \\
\hline \multirow{2}{*}{$\begin{array}{r}\text { History of egg and eggshell } \\
\text { intake }\end{array}$} & Egg, \% & $79(64.8)$ & $79(61.7)$ \\
\hline & Eggshell (powder or any other form), \% & $\mathbf{0}(\mathbf{0})$ & $\mathbf{0}(\mathbf{0})$ \\
\hline \multicolumn{4}{|c|}{ Farming and Livestock Production } \\
\hline \multirow[t]{6}{*}{ Crop production } & Cereals, \% & $\mathbf{9 7 . 5 \%}$ & $99.2 \%$ \\
\hline & Pulses, \% & $69.7 \%$ & $71.9 \%$ \\
\hline & Coffee, $\%$ & $13.9 \%$ & $14.1 \%$ \\
\hline & Chat*, \% & $37.7 \%$ & $74.2 \%$ \\
\hline & Chili*, \% & $0 \%$ & $13.3 \%$ \\
\hline & Fruits and vegetables, \% & $99(81.1)$ & $92(71.9)$ \\
\hline \multirow[t]{8}{*}{ Livestock Production } & Any livestock, $\%$ & $102(83.6)$ & $94(73.4)$ \\
\hline & Cow, \% & $72(59)$ & $62(48.4)$ \\
\hline & Ox, \% & $66(54.1)$ & $65(50.8)$ \\
\hline & Sheep, \% & $24(19.7)$ & $37(28.9)$ \\
\hline & Goat*, \% & $60(49.2)$ & $37(28.9)$ \\
\hline & Horse, \% & $1(0.8)$ & $2(1.6)$ \\
\hline & Donkey, \% & $73(59.8)$ & $63(49.2)$ \\
\hline & Chicken, \% & $46(37.7)$ & $63(49.2)$ \\
\hline
\end{tabular}

*Statistically significant, $p$-value $<0.05$ 
Baseline characteristics were comparable between the groups as shown in Table 1.Almost all caregivers were in both groups $(99.2 \%)$ were biological mothers. More than $95 \%$ of them were farmers. Illiteracy accounted for $75 \%$. About half of them had ever received education on feeding young children with eggs but no one was educated about ESP. Intervention group was comprised of a significantly higher number of younger children with 6-8 months of age compared to the control. About three-fourths of the households had two or three under five children. More than $63 \%$ of the child had a history of egg intake, but none had ever taken ESP. In both groups, cereals mainly maize, sorghum, and millet are predominantly produced followed by pulses (mainly haricot bean). Kale was the major vegetable produced in both groups. Livestock is common and $44 \%$ of the households had poultry.

Baseline child feeding practices of the two groups were similar as well. Almost all the children in both kebeles $(99.2 \%)$ were found to be on breast feeding. At the start of the study, $80.3 \%$ and $87.5 \%$ of the children were introduced to complementary foods at around six months, $6.6 \%$ and $3.1 \%$ were lately introduced, and $13.1 \%$ and $9.4 \%$ did not start foods at all other than breast milk in the intervention and control groups respectively. With no significant difference in food types consumed by the children among the groups, cereals of maize, sorghum, and millet were the most common food items $(86 \%-91 \%)$ consumed every day mainly in the form of porridge and bread ('kita'). Next were eggs followed by pulses and cow milk as well as fruits and vegetables that were consumed by about $45 \%-51 \%, 35 \%-40 \%$ and $20 \%$ $25 \%$ of the children, respectively, at least once in a month. None of them ever ate meat, fish, and chicken and about $37 \%$ had never been fed with egg.

Both study groups were comparable at baseline in anthropometric indices of mean WAZ $(\mathrm{p}=.650)$ and MUACZ scores $(p=749)$. However, the intervention arm had better LAZ and WHZ scores with significant differences compared to the control. At endline, children in the intervention arm had better anthropometry outcomes when compared to control (Table 2). Particularly, WAZ significantly increased ( $\beta=0.24)$ in the Intervention group after adjusting for child age and baseline measure. No significant differences were observed in underweight and stunting rates of the children in the intervention and control groups at baseline. After six months of intervention, underweight $(\mathrm{p}=.020)$, stunting $(\mathrm{p}=.045)$ and wasting rates $(\mathrm{p}=.008)$ showed Stratified by baseline anemia status, the post-intervention analysis showed that stunting was increased in both anemic $(n=89)$ and nonanemic $(n=161)$ child population of Intervention and Control groups at endline. However, the increment was less by $28.3 \%$ in the non-anemic children of the treatment group $(n=84)$ than that of the control arm $(n=77)$. Similar analysis also showed that underweight was decreased by $65.1 \%$ in the non-anemic children population of the treatment group than that of the control arm. In addition, stratified analysis by age group revealed that underweight was significantly decreased by $54.3 \%$ in children of 6-9 months age group of the intervention group $(n=69)$ when compared to the same age group in the control arm $(n=63)$, as shown in Table 2.

Trend analysis showed that underweight rate demonstrated an increasing trend in both groups from May to June 2016, the period when implementation of intervention activities was to be started. After June 2016, the trend showed a significant difference $(\mathrm{p}=.020)$ between the groups that underweight

decreased in the chicken group from $19 \%$ to $17 \%$ while increased in the control from $28 \%$ to $30 \%$. The difference between the groups was more visible in the trend of severe underweight rate that decreased from 5\% (in June 2016) to $0.8 \%$ at the end of intervention (Nov 2016) in the intervention group and, to the contrary, increased in the control from $8.6 \%$ to $10.2 \%$ (Figure 2 ).

Stunting $(<-2$ SD LAZ) showed an increasing trend in both groups with a slightly lower slope in the intervention arm. Severe stunting $(<-3$ SD LAZ) was also seen increasing among the young children of both groups; however, it increased at a visibly slower rate or slope in the intervention group (Figure 3). Severe stunting rate increased by more than three folds in the young children of the control group (from $6.3 \%$ to $21.1 \%$ ) while it increased by two-fold in the intervention arm (from 5.2\% to $10.7 \%$ ).

Intervention group had significantly higher hemoglobin levels at baseline compared to the control $(p=.016)$. At endline, mean hemoglobin was increased remarkably in both study groups maintaining the significant difference between the groups observed at baseline. Egg and ESP interventionincreased haemoglobin levels after adjusting for age and baseline anemia status (Table 2). Prevalence of anemia $(\mathrm{Hb}<11.0 \mathrm{~g} / \mathrm{dL})$ was $31.1 \%$ and $39.8 \%$ at baseline and $18 \%$ and $28.9 \%$ at endline among the children in the intervention and control groups respectively. The groups had comparable anemia prevalence at baseline $(\mathrm{p}=.151)$; however, it showed a significant difference after six months of intervention ( $\mathrm{p}=.043)$. Based on WHO 
anemia classification by $\mathrm{Hb}$ level[11], $19.7 \%$ and $11.5 \%$ of the children in the intervention and $25 \%$ and $14.8 \%$ in the control group were mildly $(\mathrm{Hb}=10-10.9 \mathrm{~g} / \mathrm{dL})$ and moderately $(\mathrm{Hb}=7-9.9 \mathrm{~g} / \mathrm{dL})$ anemic respectively at baseline. This magnitude decreased to $10.7 \%$ and $7.4 \%$ in the intervention and $18.8 \%$ and $10.2 \%$ in the control arm as well in the respective order.
The total anemia prevalence was reduced by $42.1 \%$ and $27.4 \%$ in the intervention and control groups respectively. Stratified analysis revealed that, after the intervention, anemia was significantly decreased by $52.2 \%$ in children of $9-12$ months age group of the intervention group when compared to that of the control arm. This age group had longer ESP intake (for about four and half months) compared to children of 6-8 months who consumed ESP for one and half month on average when ESP intake was started at one year of age.

Table 2: Effect of egg and eggshell powder (ESP) consumption with a 6-month Chicken Intervention on effect size and relative risk (RR) of nutritional and anaemia status of young children in Southern Ethiopia District, 2016.

\begin{tabular}{|c|c|c|c|c|c|c|c|c|}
\hline & \multicolumn{3}{|c|}{ Baseline } & \multirow{2}{*}{$\begin{array}{l}\text { End line } \\
\begin{array}{l}\text { Control } \\
(\mathrm{n}=128)\end{array} \\
\end{array}$} & \multicolumn{2}{|c|}{$\begin{array}{c}\text { Effect size ( } \beta) \\
\text { and RR }\end{array}$} & \multicolumn{2}{|c|}{$\begin{array}{c}\text { Effect size ( } \beta \text { ) } \\
\text { and RR }\end{array}$} \\
\hline & $\begin{array}{c}\text { Intervention } \\
(\mathrm{n}=122)\end{array}$ & $\begin{array}{l}\text { Control } \\
(\mathrm{n}=128)\end{array}$ & $\begin{array}{c}\text { Intervention } \\
(\mathrm{n}=122)\end{array}$ & & \multicolumn{2}{|c|}{ Unadjusted } & \multicolumn{2}{|c|}{ Adjusted } \\
\hline & Mean (SD) & Mean (SD) & Mean (SD) & Mean (SD) & $\beta(95 \% \mathrm{CI})$ & $\mathbf{P}$ & $\beta(95 \% \mathrm{CI})$ & $\mathbf{P}$ \\
\hline LAZ & $-1.26(1.09)$ & $-1.56(1.09)$ & $-1.86(0.99)$ & $-2.13(1.01)$ & $0.23(0.06-0.55)$ & .020 & $0.06(-0.06-0.19)^{\mathrm{a}}$ & .327 \\
\hline WAZ & $-1.11(1.09)$ & $-1.37(1.10)$ & $-1.07(0.96)$ & $-1.50(1.08)$ & $0.43(0.18-0.69)$ & .001 & $0.24(0.11-0.37)^{\mathrm{a}}$ & $<001$ \\
\hline wHz & $-0.09(0.96)$ & $-0.36(0.97)$ & $-0.25(0.95)$ & $-0.63(1.02)$ & $0.38(0.14-0.63)$ & .002 & $0.21(0.06-0.37)^{a}$ & .008 \\
\hline \multirow[t]{2}{*}{ Hemoglobin } & $11.50(1.22)$ & $11.14(1.13)$ & $11.75(1.15)$ & 11.40 (1.12) & $0.35(0.06-0.63)$ & .017 & $0.31(0.03-0.55)^{\mathrm{b}}$ & .032 \\
\hline & $(\%)$ & $(\%)$ & $(\%)$ & $(\%)$ & $\mathrm{RR}(95 \% \mathrm{CI})$ & $\mathbf{P}$ & $\mathrm{RR}(95 \% \mathrm{CI})$ & $\mathbf{P}$ \\
\hline \multirow{2}{*}{ Stunting } & \multirow{2}{*}{$31(25.4)$} & \multirow{2}{*}{$46(35.9)$} & \multirow{2}{*}{$56(45.9)$} & \multirow{2}{*}{$75(58.6)$} & \multirow{2}{*}{$0.78(0.62-1.00)$} & \multirow{2}{*}{.045} & $0.74(0.53-1.04)^{c}$ & .080 \\
\hline & & & & & & & $0.72(0.53-0.98)^{d}$ & .032 \\
\hline \multirow{2}{*}{ Underweight } & \multirow{2}{*}{$23(18.9)$} & \multirow{2}{*}{$36(28.1)$} & \multirow{2}{*}{$21(17.2)$} & \multirow{2}{*}{$38(29.7)$} & \multirow{2}{*}{$0.58(0.36-0.93)$} & \multirow{2}{*}{.020} & $0.46(0.25-0.83)^{c}$ & .008 \\
\hline & & & & & & & $0.35(0.16-0.74)^{d}$ & .003 \\
\hline Anemia & $38(31.1)$ & $51(39.8)$ & $22(18.0)$ & $37(28.9)$ & $0.62(0.39-0.99)$ & .043 & $0.48(0.24-0.96)^{\mathrm{e}}$ & .030 \\
\hline
\end{tabular}

Multiple linear regression model for unadjusted and adjusted effect size of anthropometry and hemoglobin measures as well as stratified analysis and cross tabulation for risk estimation of nutrition and anemia outcomes.

a LAZ, WAZ and WHZ were adjusted for child age and baseline anthropometry for the same dependent variable. b Hb was adjusted for age as well as anemia status at baseline. c Adjusted for age (age group of 6-9 and 10-12 months). d Adjusted for baseline anemia status. e Adjusted for age (age group of 6-8 and 9-12 
Anteneh Omer et al.

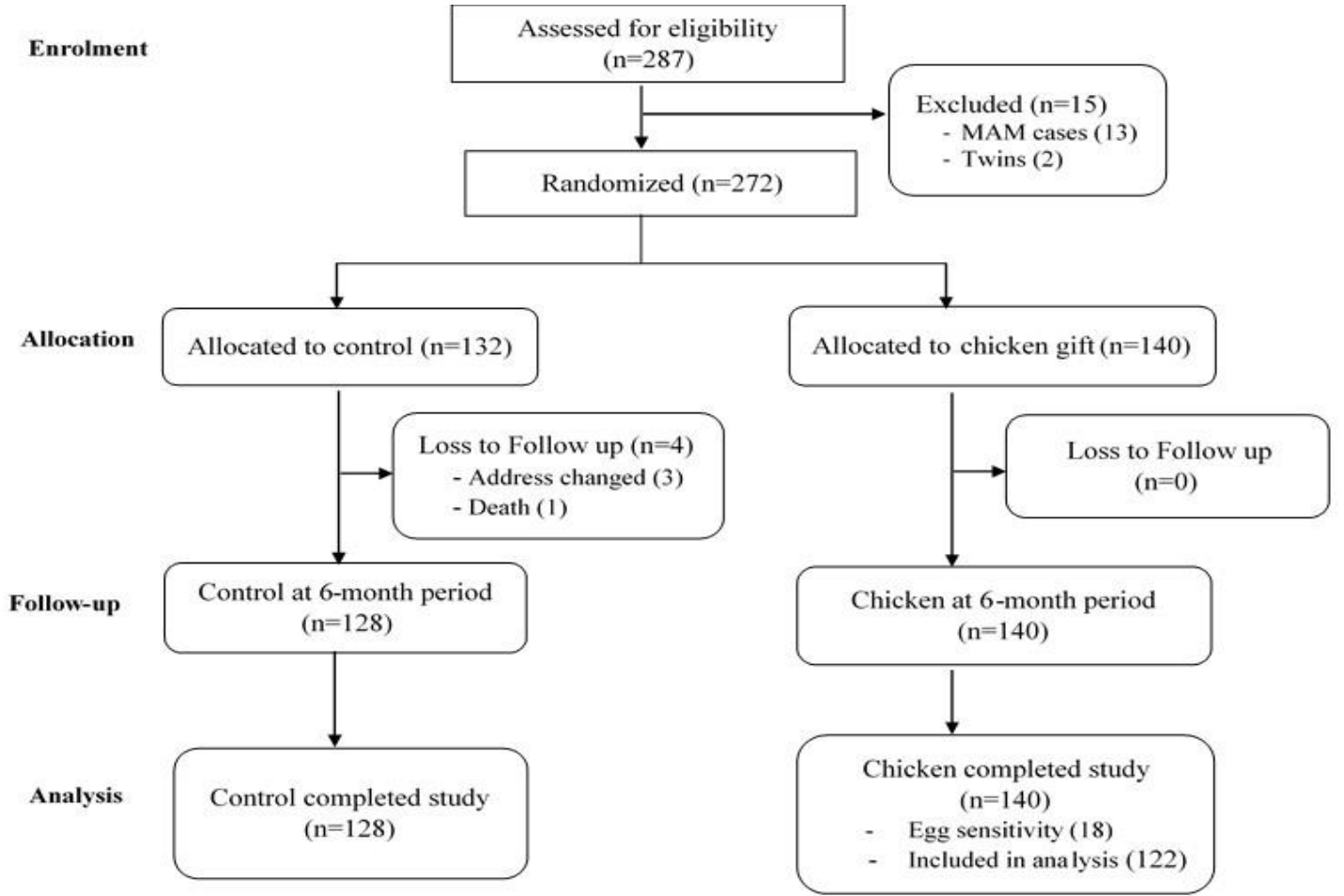

Figure 1: Flow diagram showing recruitment

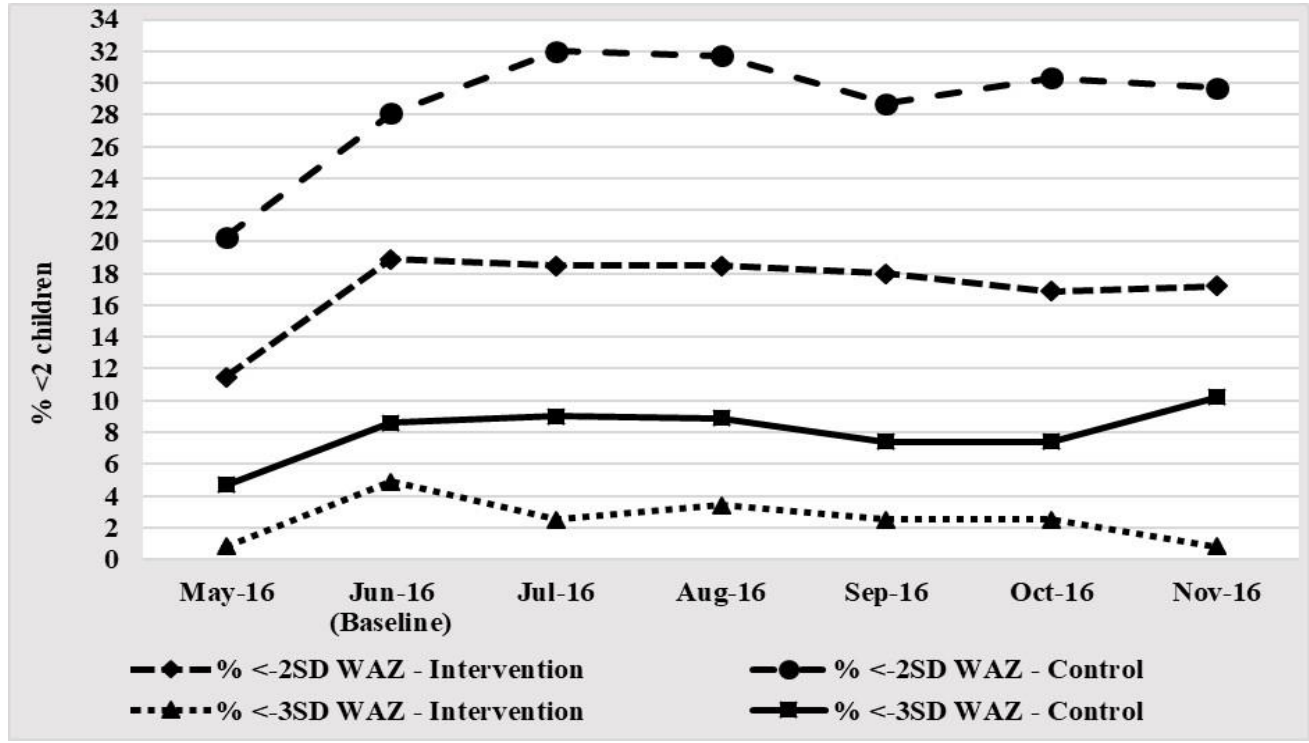

Figure 2: Trend of underweight among Intervention and Control groups 
Anteneh Omer et al.

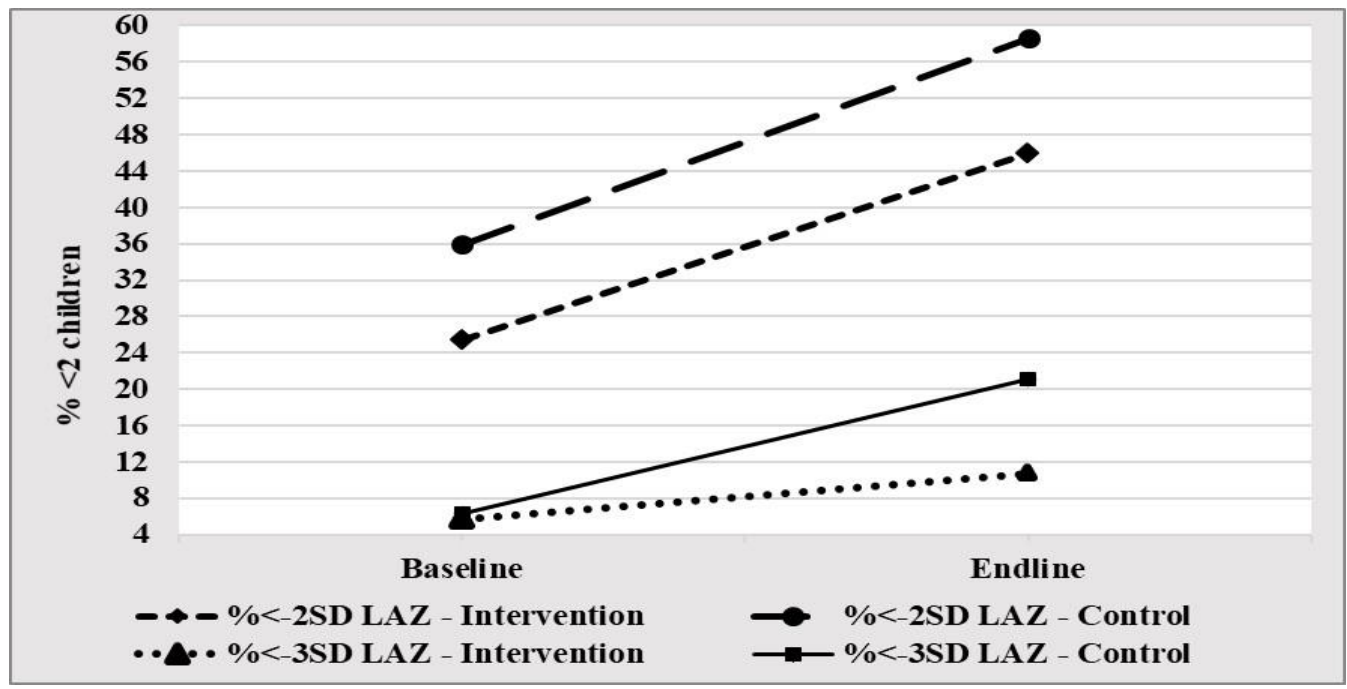

Figure 3: Trend of stunting among Intervention and Control groups

Diarrhea, fever, vomiting, and cough were the most commonly reported symptoms of morbidity in both groups. The young children in both groups were not significantly different in their morbidity status at baseline $(\mathrm{p}=0.479)$. Egg and ESP intervention did not show any effect on the reduction of morbidity symptoms and total illness $(\mathrm{p}=0.159$, $95 \% \mathrm{CI}=-0.04-0.21)$ ) when compared to the control. Paired t-test revealed that total illness as a whole and diarrhea, in particular, were significantly reduced in both groups at the end of the intervention. No actual signs and symptoms of egg allergy were observed in any children. However, 18 children (10 males and 8 females) in the intervention group were found to be sensitive to eggs. This accounts for $7.5 \%$ of the total children included in the intervention group $(n=140)$. Egg sensitivity was seen in all ages ranging from 6-11 months. The earliest report of sensitivity was received after two months of egg introduction. Among the egg sensitive children, eight $(44.4 \%)$ refused to eat eggs. The remaining ate small amounts of an egg despite their sensitivity. The reaction to egg was expressed by vomiting the egg and other foods eaten together with the egg. The children showed their unwillingness to eat eggs by different signs including spitting out $(44.4 \%)$, turning their face away (16.7\%), trying to escape/run away (27.8\%) and pushing the plate (5.6\%). Caregivers tried different ways to solve the problem of egg sensitivity by feeding egg every other day $(83.3 \%)$, feeding egg mixed with other foods like boiled and smashed potato (38.9\%), stop feeding egg temporarily (16.7\%) and feeding fried egg (5.6\%). Stopping intake of egg for these children resolved the situation completely.

\section{Discussion}

In Sub-Saharan Africa, zinc, iron and calcium are the major limiting nutrients in the complementary diets of young children [12]; eggs and ESP may fill those dietary gaps. In this 6-month controlled community trial, we found that increased egg and ESP intake had a positive and significant effect on improved health and nutrition outcomes of young children under two years of age. The largest effects were seen on increasing WAZ score and a subsequent reduction of underweight as well as anaemia in the intervention group when compared to the control arm. Both intervention and control groups had a similar increasing trend of the underweight rate during the pre-intervention period from May to June 2016. This is the natural and expected trend of underweight as it was the "lean" season from May to June when household food stores get depleted and food insecurity becomes worse [13]. Despite household food scarcity during the lean season, consumption of eggs and ESP appeared to have protected the children from being underweight. The introduction of the egg at an age of 6-9 months (egg yolk at 6-7 months and afterward whole egg) was found to be effective in reduction of underweight. This is the period when children start feeding on different weaning foods in addition to breast milk. Moreover, nutritional status gets to deteriorate due to poor feeding practice, poor hygienic situation, and recurrent illnesses. The significant reduction in underweight $(54 \%)$ observed in this age group indicates the potential of adding just one egg a day in the children's diet at an early age in the prevention 
and reduction of undernutrition before it occurs or gets worse and difficult to reverse [8].

A recent study provided evidence that ingestion of animal source protein showed an improvement in height-for-age in children aged 12-36 months in Malawi [14]. In our study, consumption of egg and ESP had no apparent effect on increasing length for age. However, this intervention showed a modest $(\mathrm{P}<0.05)$ positive effect on stunting reduction. Both study groups had comparable stunting prevalence at baseline that escalated above the national figures at endline in both arms showing significant group difference. Stunting increased at a slower rate in the intervention group compared to that of the control. It did not increase in the treatment group to the level it should have reached following the trend of the control group which might be due to the increased egg and ESP intake. Stunting and anemia are now thought to be related [15]. After we adjusted for baseline anemia status, children in the treatment group were found to be $28 \%$ less likely to get stunted at endline when compared to controls. It is possible that the effect on stunting reduction would have been greater and more significant if the chickens had provided eggs earlier in the full 6-month time period [9]. What was achieved is the effect of four months of every other day egg and, when children were over 1 year of age, ESP consumption. An egg-a-day trial in Eucador reported $74 \%$ and $47 \%$ reduction of underweight and stunting, respectively, wherein children of 6-9 months old received eggs for daily intake for six months [8]. No other comparable intervention study was found in young children of resource-poor populations. One observational study reported an inconsistent effect of increased egg consumption on underweight in different rural villages of Sub-Saharan Africa [16]. A large scale study in India associated lower consumption of eggs with a two-fold increased odds of stunting in under two children [17].

Evidence for the effect of egg/ESP intake on hemoglobin and anaemia are limited. A comparable result was reported by a study in rural China that encouraged caregivers to give hardboiled egg yolk to infants daily after the age of 4 months. Caregivers in the intervention group were much better in feeding their children with egg yolk that resulted in significantly increased mean hemoglobin level and reduced anemia prevalence compared with those in the control group [18].

Being a predictor of underweight and stunting [19], underlying anemia was seen in this study confounding the effect of egg/ESP intake on reducing underweight and stunting in young children. Our findings suggest that foodbased interventions need to be integrated with anemia prevention and treatment strategies in order to bring significant change in reduction of underweight particularly incountries like Ethiopia where anemia is significant public health problem.

Calcium intake may negatively affect iron absorption, yet long-term $\mathrm{Ca}$ supplementation has failed to show any adverse effects on Fe status, indicating the effect of $\mathrm{Ca}$ on $\mathrm{Fe}$ absorption may be of short duration and adaptation may occur with time [20]. In our study, the age group of 9-12 months that had ESP consumption, showed a marked reduction of anemia. This result seems supporting the findings of long-term calcium intake has an insignificant effect on iron absorption. Further investigation is needed to elucidate the effect of ESP on the iron status of young children and its public health impact on anemia reduction.

Morbidity in general and diarrhea, in particular, were reduced significantly in both intervention and control groups, but with no group difference. Cough, fever, and vomiting did not show a significant reduction in both groups and no group difference. Though not significant, vomiting was less frequent in the control group compared with the intervention arm. It might be due to egg sensitivity. In agreement with this study, Iannotti and co-workers [8] reported that egg intervention had no significant effect on morbidity reduction. However, there was an increased report of acute diarrhea in that study within the treatment group which was not the case in our study. Food allergy is one of the commonly mentioned challenges of egg interventions. Signs and symptoms of actual egg allergy were not seen in any of the children similar to the Ecuador egg trial [8]. Delayed introduction of egg because of concerns about allergy has recently been revised to introduce at an early age to reduce the likelihood of sensitization to egg proteins and thereby prevent the development of allergy [21]. In this study, less than $8 \%$ of the children showed sensitivity reaction by vomiting the egg and other foods what they already ate. Egg sensitivity was seen in both sexes and all ages. The reaction occurred, as reported by caregivers, the earliest two months after eggs were introduced to the children. Feeding eggs mixed with other foods like boiled and smashed potato every other day helped resolve the sensitivity as reported by caregivers. There are limitations of the study. The follow-up period of this study was only six months which was further reduced by the fact that chickens did not lay sufficient eggs for the first 2 months of the study [9]. Results of this study are generally the combined effects of egg and ESP consumption on the nutrition 
status of young children; however, ESPwas not given to all children for the same length of time as the egg. Precautions were takenduring random selection and allocation of study kebeles so that there was no information sharing among the study kebeles. Although the randomization process generated comparable groups on all other observed characteristics, there were baseline differences in anthropometric and hemoglobin measures between the intervention and control groups. These differences were accounted by adjusting for baseline anthropometric and hemoglobin measures in all analysis.

\section{Conclusion}

This community trial demonstrated that increased egg and ESP intake significantly reduced underweight and anemia among infants of rural Halaba population. Our study is in agreement with the view that eggs have important nutritional benefit for the at-risk pediatric population in low-income countries where ASFs are not readily available. Our study showed that children benefited through the reduction in underweight, stunting, and anemia in a homestead poultry operation that stressed child ownership. The results of this trial can be used to strengthen ongoing efforts of poultry interventions focusing on child nutrition in Ethiopia and other countries where ASF intake is low and the burden of undernutrition is high.

\section{Acknowledgments}

Primary funding for this study was provided by One Health, University of Saskatchewan. A grant from the Saskatchewan Egg Producers was used to offset cost of the chickens.

\section{Conflict of interest}

The authors declare no conflict of interests.

\section{Reference}

1. Central Statistical Agency (CSA) [Ethiopia] and ICF. Ethiopia Demographic and Health Survey 2016. Addis Ababa, Ethiopia, and Rockville, Maryland, USA: CSA and ICF.

2. Ethiopian Public Health Institute. Ethiopian National Food Consumption Survey 2013. Addis Ababa, Ethiopia

3. Iannotti LL, Lutter CK, Bunn DA, Stewart CP. Eggs: the uncracked potential for improving maternal and young child nutrition among the world's poor. Nutr Rev. 2014; 72(6):355-368.

4. Daengprok W, Garnjanagoonchorn W, Naivikul O, Pornsinlpatip P, Issigonis K, Mine Y. Chicken eggshell matrix proteins enhance calcium transport in the human intestinal epithelial cells, Caco-2. J Agric Food Chem. 2003; 51(20): 6056-6061.
5. Brun LR, Lupo M, Delorenzi DA, Di Loreto VE, Rigalli A. Chicken eggshell as suitable calcium source at home. Int J Food Sci Nutr. 2013; 64(6):740-743.

6. Dumas SE, Lungu L, Mulambya N, Daka W, Lewis D, Travis A. Effect of sustainable poultry interventions on household food security and resilience in game management areas of Zambia's Luangwa Valley: a before-and-after study. Lancet Glob Health. 2017; 5: S24.

7. Atuobi-Yeboah A, Marquis GS, Colecraft E, Kanlisi R, Aryeetey R, Klevor M. An integrated nutrition-sensitive health and agriculture intervention to increase egg consumption among infants and young children in Upper Manya Krobo, Ghana. FASEB J. 2016;30 (1_supplement):274.5.

8. Iannotti LL, Lutter CK, Stewart CP, et al. Eggs in early complementary feeding and child growth: a randomized controlled trial. Pediatrics. 2017; 140(1):e20163459.

9. Omer A, Mulualem D, Classen H, Vatanparast $\mathrm{H}$, Whiting SJ. A community poultry intervention to promote egg and eggshell powder consumption by young children in Halaba Special Woreda, SNNPR, Ethiopia. J Agric Sci. 2018; 10(5):1.

10. Dewey K, Adu-Afarwuah S. Systematic review of the efficacy and effectiveness of complementary feeding interventions in developing countries. Matern Child Nutr. 2008;4: 24-85.

11. WHO. Haemoglobin concentrations for the diagnosis of anaemia and assessment of severity. Vitamin and Mineral nutrition information system. 2011. Geneva, World Health Organization, (WHO/NMH/NHD/MNM/11.1).

12. Ferguson E, Chege P, Kimiywe J, Wiesmann D, Hotz C. Zinc, iron and calcium are major limiting nutrients in the complementary diets of rural Kenyan children: TBA training improves early infant feeding. Matern Child Nutr. 2015; 11:6-20. doi:10.1111/mcn.12243

13. Hirvonen K, Taffesse AS, Worku Hassen I. Seasonality and household diets in Ethiopia. Public Health Nutr. 2016; 19(10):1723-1730.

14. Kaimila Y, Divala O, Agapova SE, et al. Consumption of animal-source protein is associated with improved height-for-age $\mathrm{z}$ scores in rural Malawian children aged 12-36 months. Nutrients. 2019; 11, 480. 
15. Mohammed SH, Larijani B and Esmaillzadeh A. Concurrent anemia and stunting in young children: prevalence, dietary and nondietary associated factors. Nutr J. 2019; 18:10.

16. Hetherington JB, Wiethoelter AK, Negin J, Mor SM. Livestock ownership, animal source foods and child nutritional outcomes in seven rural village clusters in Sub-Saharan Africa. Agric Food Secur. 2017;6(1):9.

17. Aguayo VM, Nair R, Badgaiyan N, Krishna V. Determinants of stunting and poor linear growth in children under 2 years of age in India: an in-depth analysis of Maharashtra's comprehensive nutrition survey: Child stunting in Maharashtra, India. Matern Child Nutr. 2016;12:121-140. doi:10.1111/mcn.12259

18. Guldan GS, et al. culturally appropriate nutrition education improves infant feeding and growth in Rural Sichuan, China. J Nutr. 2000; 1204-1211.

19. Thorne CJ, et al. Anaemia and malnutrition in children aged 0-59 months on the Bijagós Archipelago, GuineaBissau, West Africa: a cross-sectional, population-based study. Paediatr Int Child Health. 2013; 33(3):151-160.

20. Lönnerdal B. Calcium and iron absorption--mechanisms and public health relevance. Int J Vitam Nutr Res. 2010 Oct;80(4-5):293-299.

21. Yalçin S.S. \& Yalçin S. Poultry eggs and child health - a review. Lohmann Information. 2013;48 (1), 3-14. 